CLINICAL STUDY

\title{
Increased $N \varepsilon$-(carboxymethyl)-lysine levels in cerebral blood vessels of diabetic patients and in a (streptozotocin-treated) rat model of diabetes mellitus
}

\author{
A W van Deutekom ${ }^{1}$, H W M Niessen ${ }^{2,3,4}$, C G Schalkwijk ${ }^{5}$, R J Heine ${ }^{1}$ and S Simsek ${ }^{1}$ \\ ${ }^{1}$ From the Department of Endocrinology/Diabetes Center, ${ }^{2}$ Department of Pathology, ${ }^{3}$ Department of Cardiac Surgery and ${ }^{4}$ Institute for Cardiovascular \\ Research (ICAR-VU), VU University Medical Center, PO Box 7057, 1007 MB, Boelelaan 1117, Amsterdam, The Netherlands and ${ }^{5}$ Department of \\ Internal Medicine, Maastricht University Hospital, Debeyelaan 25, PO Box 5800, 6202 AZ, Maastricht, The Netherlands \\ (Correspondence should be addressed to S Simsek; Email: simsek@vumc.nl)
}

\begin{abstract}
Objective: Non-enzymatic glycation of proteins and their end products (advanced glycation end products, AGE) have been implicated in the pathogenesis of diabetic complications. Our aim was to evaluate the association between diabetes mellitus (DM) and the accumulation of one of the most abundant AGEs, NE-(carboxymethyl)-lysine (CML), in cerebral vessels.

Research design and methods: Brain tissue samples were obtained by autopsy from 20 DM patients and 13 age-matched controls. In addition, we investigated brain tissue samples of seven rats after induction of diabetes with streptozotocin (STZ) and six non-diabetic control rats. We used an immunohistochemical staining method to examine the CML immunoreactivity in the cerebral vessels.

Results: Staining intensity of CML was significantly higher in cerebral vessels of diabetic patients than in non-diabetic subjects (median of the immunohistochemical intensity score $/ \mathrm{cm}^{2}$ in the diabetic group of 0.85 (interquartile range (IQR) $0.66-1.52$ ) vs 0.63 in the control group (IQR $0.44-0.70$ ); $P=0.002)$. Furthermore, there was a similar significant difference in CML staining intensity of cerebral vessels between STZ diabetic rats and non-diabetic control rats (median of the immunohistochemical intensity score $/ \mathrm{cm}^{2}$ in the diabetic group of 1.08 (IQR 0.73-1.43) vs $0.23 \mathrm{in}$ the control group (IQR 0.12-0.43); $P=0.003$ ).

Conclusions: Accumulation of CML-modified proteins is significantly greater in the cerebral vessels of the diabetic patients than their age-matched controls. This association has been confirmed in the insulin-deficient diabetic rat model. It may be possible that the excessive accumulation of AGE-modified proteins in the cerebral vasculature alters the local environment and microcirculation and thereby contributes to the development of cognitive impairments in diabetes. Therefore, additional study on the causal link between AGE accumulation and cognitive dysfunction and the potential benefits of AGEblocking and/or breaking compounds is indicated.
\end{abstract}

European Journal of Endocrinology 158 655-660

\section{Introduction}

Prolonged exposure to chronic hyperglycaemia triggers abnormalities resulting in the occurrence of welldescribed diabetic complications, including nephropathy, retinopathy and peripheral neuropathy (1). Besides these long-term complications, diabetes mellitus (DM) can also affect the brain function. This may ultimately lead to a state of cognitive impairment, in particular, psychomotor slowing and reduced mental flexibility (2). In addition to this diabetes-associated cognitive decline (DACD) (3), DM increases the risk of dementia, cerebral atrophy and leukoencephalopathy not directly attributed to infarction (4). In recent years, it has become increasingly clear that vascular dysfunction plays a crucial role in the manifestation of long-term diabetic complications. It is believed that chronic hyperglycaemia influences endothelial cell function and thereby causes vascular derangements. The precise mechanisms that link hyperglycaemia to vascular disease have not yet been established, although several hypotheses have been proposed. One such hypothesis focuses on non-enzymatic glycation and oxidation, suggesting that glycoxidation reactions and oxidative stress are important factors in the pathogenesis of vascular dysfunction and diabetic complications (5). In this process, amino groups in proteins, lipids and nucleic acids exposed to reducing sugars undergo a series of molecular rearrangements, resulting in the irreversible formation of a heterogeneous class of sugarderived adducts. As a group, the late products of this cascade have been termed advanced glycation end 
products (AGEs) (5). One of the most abundant and best characterized AGE is $\mathrm{N \varepsilon}$-(carboxymethyl)-lysine (CML) (6) that can also be formed from oxidation of free fatty acids. AGE formation occurs during normal ageing, but is enhanced in DM, as a result of the associated hyperglycaemia (6). Since the formation of CML is irreversible, it has been suggested that CML may provide indirect evidence for the cumulative oxidative damage to the respective tissue. Of particular interest is the finding that CML levels in tissue and serum are related with the severity of several diabetic complications $(7,8)$.

In an attempt to elucidate whether the AGE accumulation in the cerebral vessels of diabetic patients is significantly higher than in non-diabetic individuals, we investigated the immunohistochemical distribution of CML in post-mortem brain tissue from subjects with DM and age-matched controls. The study was extended by studying the CML distribution in the brains of hyperglycaemic streptozotocin (STZ)-treated diabetic rats and normoglycaemic rats as controls.

\section{Materials and methods}

\section{Subjects}

Representative brain tissue samples from the cortex (one region per patient) were obtained by autopsy from 20 DM patients and 13 age-matched controls. None of the controls had a clinical history of DM or elevated blood glucose levels. The mean ages of the DM cases and the controls were 61.9 years (range 9-86 years) and 66.9 years (range 7-92 years) respectively. The DM cases and controls were clinically and historically without neurological disorders, including acute disorders such as cerebrovascular events and brain oedema caused by head injury. Details of the patients are given in Table 1 .

\section{Animal model}

Two groups of adult male Wistar rats (Charles River, Maastricht, The Netherlands), weighing $\sim 250 \mathrm{~g}$, were used (as described previously, (9)), namely a control group $(n=6)$ and a diabetic group $(n=7)$. Diabetes was induced by a single i.p. administration of STZ ( $60 \mathrm{mg} / \mathrm{kg}$; Sigma). STZ diabetes was confirmed by measuring the blood glucose level 3 days after the induction. Diabetes was verified by a serum glucose level $>13.9 \mathrm{mmol} / \mathrm{l}$. After 6 weeks of STZ diabetes, animals were killed by decapitation, followed by harvesting of brain tissues. In the end, we obtained brain tissue from seven diabetic rats and six nondiabetic controls.

The protocol was performed in compliance with the European Community guidelines for the use of experimental animals and approved by the National Authorities for Animal Experiments.
Table 1 Characteristics of study subjects.

\begin{tabular}{|c|c|c|c|}
\hline Age (years) & $\begin{array}{l}\text { Type of } \\
\text { DM }\end{array}$ & $\begin{array}{l}\text { Clinical diagnosis, } \\
\text { cause of death }\end{array}$ & $\begin{array}{c}\text { Brain } \\
\text { weight }(\mathrm{g})\end{array}$ \\
\hline \multicolumn{4}{|c|}{ DM patients $(n=20)$} \\
\hline 9 & 1 & Arrhythmia & 1342 \\
\hline 22 & 1 & DKA & 1470 \\
\hline 25 & 1 & Opioid abuse & 1500 \\
\hline 25 & 1 & Arrhythmia & 1500 \\
\hline 41 & 1 & CVA & 1510 \\
\hline 52 & 2 & CMV pneumonia, ARDS & 1690 \\
\hline 55 & 2 & $\begin{array}{l}\text { Acute renal failure, } \\
\text { myocardial infarction }\end{array}$ & 1146 \\
\hline 59 & 2 & Myocardial infarction & 1133 \\
\hline 67 & 2 & Ruptured AAA & 1120 \\
\hline 69 & 2 & $\begin{array}{l}\text { Pericarditis, broncho- } \\
\text { pneumonia }\end{array}$ & 1475 \\
\hline 73 & 2 & Myocardial infarction & 1530 \\
\hline 76 & 2 & $\begin{array}{l}\text { Chronic B-lymphocytic } \\
\text { leukaemia, pneumo- } \\
\text { nia }\end{array}$ & 1355 \\
\hline 78 & 2 & $\begin{array}{l}\text { Adenocarcinoma of } \\
\text { the lung, broncho- } \\
\text { pneumonia }\end{array}$ & 1457 \\
\hline 81 & 2 & Myocardial infarction & 1360 \\
\hline 81 & 2 & Pneumonia, sepsis & 1290 \\
\hline 84 & 2 & Myocardial infarction & 1225 \\
\hline 84 & 2 & $\begin{array}{l}\text { Pulmonary thrombo- } \\
\text { embolism }\end{array}$ & 1220 \\
\hline 85 & 2 & Pneumonia, sepsis & 1230 \\
\hline 86 & 2 & Sepsis & 1115 \\
\hline & 2 & Bronchopneumonia & 1100 \\
\hline \multicolumn{4}{|c|}{ Controls $(n=13)$} \\
\hline 7 & & Arrhythmia & 1157 \\
\hline 40 & & $\begin{array}{l}\text { Small cell carcinoma, } \\
\text { bronchopneumonia }\end{array}$ & 1279 \\
\hline 55 & & $\begin{array}{l}\text { Small cell carcinoma, } \\
\text { bronchopneumonia }\end{array}$ & 1650 \\
\hline 59 & & $\begin{array}{l}\text { Chronic alcohol abuse, } \\
\text { hepatic insufficiency }\end{array}$ & 1064 \\
\hline 66 & & Sepsis & 1401 \\
\hline 68 & & $\begin{array}{l}\text { Hypopharynx carci- } \\
\text { noma, rupture of } \\
\text { common carotid artery }\end{array}$ & 1450 \\
\hline 72 & & $\begin{array}{l}\text { Adenocarcinoma of the } \\
\text { lung, broncho- } \\
\text { pneumonia }\end{array}$ & 1515 \\
\hline 83 & & Myocardial infarction & 1182 \\
\hline 87 & & $\begin{array}{l}\text { Pancreatic ductal carci- } \\
\text { noma, broncho- } \\
\text { pneumonia }\end{array}$ & 1190 \\
\hline 92 & & Sepsis & 1193 \\
\hline 73 & & Myocardial infarction & 1356 \\
\hline 78 & & Myocardial infarction & 1297 \\
\hline 89 & & $\begin{array}{l}\text { Bronchopneumonia, } \\
\text { myocardial infarction }\end{array}$ & 1324 \\
\hline
\end{tabular}

DM, diabetes mellitus; DKA, diabetic ketoacidosis; CVA cerebrovascular accident; ARDS, acute respiratory distress syndrome; CMV, cytomegalovirus; AAA, abdominal aortic aneurysm.

\section{Immunohistochemical staining methods}

To investigate the presence and accumulation of CML in tissues, a monoclonal anti-CML antibody was used as described recently (10).

All brain tissue samples, both humans and rats, were fixed in $4-6 \%$ buffered formaldehyde, $\mathrm{pH} 7.4$, 
followed by processing and embedding in paraffin wax, as routinely performed. From each block ( $\sim 5 \mathrm{~cm}^{2}$ brain tissue), $4 \mu \mathrm{m}$ sections were cut and placed on silanized slides. Subsequently, the formalinfixed and paraffin-embedded tissue sections were deparaffinized, incubated with $0.3 \% \mathrm{H}_{2} \mathrm{O}_{2}$ in methanol for $30 \mathrm{~min}$ and enzymatically pre-treated with $0.1 \%$ pepsin. Sections were not heated to prevent artificial heat-induced CML formation (11). After washing steps with PBS ( $\mathrm{pH}$ 7.4) and further blocking by normal bovine serum (1:50; Dako, Glostrup, Denmark), sections were incubated with anti-CML (1:500, Dako) for $60 \mathrm{~min}$. After further washings, sections were incubated for 30 min with a rabbit anti-mouse biotin-labelled secondary antibody (1:500; Dako), rinsed with PBS and incubated with streptavidin-horseradish peroxidase (1:200; Dako) at room temperature for $60 \mathrm{~min}$. The colour reaction was carried out by incubating the slides with 3,3-diamino-benzidine-tetrahydrochloride $/ \mathrm{H}_{2} \mathrm{O}_{2}$ (Sigma Chemical Company) for $5 \mathrm{~min}$. After a final washing, counterstaining of the nuclei with haematoxylin was performed.

\section{Immunoscoring}

Quantification of the immunohistochemical staining was determined visually in a microscopic study. In each slide, the positively stained vessels were scored for the intensity of CML staining. Therefore, each positive vessel was given a score of 1 (meaning weak positive staining), 2 (moderate staining) or 3 (strong positive staining). Next, each intensity score was multiplied by the amount of vessels positive for this score. Each multiplication score (for 1, 2 and 3) was then added and the sum score was divided by the total slide area (in $\mathrm{cm}$ ) giving an immunohistochemical score per $\mathrm{cm}^{2}$.

\section{Statistical analysis}

Data are presented as median (interquartile range, IQR). Comparison of two study groups was performed using Mann-Whitney $U$ test. A $P$ value $<0.05$ was considered statistically significant. The relationship between age and staining intensity was evaluated using regression analysis. All analyses were performed with the statistical package SPSS 15.0 (SPSS Inc., Chicago, IL, USA).

\section{Results}

\section{Human tissue}

The presence of CML in human brain tissue, obtained from DM and non-DM patients, was investigated. Immunohistochemical analysis showed CML staining in the astrocytes (not shown) and the endothelial cells of the cerebral vasculature (Fig. 1), as described previously (12). We now focused on and quantified the intensity of the CML staining in the cerebral vessels in DM and non-DM brain tissues. None of the tissue samples showed morphological damage of the vessels or any sign of inflammation. Significant differences in the immunohistochemical intensity score of CML staining were evident between DM patients and age-matched controls (Fig. 2). The CML immunoreactivity of the cerebral vessels was significantly more intense in the DM group than in the controls (median of the immunohistochemical intensity $\mathrm{score} / \mathrm{cm}^{2}$ in the DM group of 0.85 (IQR 0.66-1.52) vs 0.63 (IQR 0.44-0.70) in the control group; $P=0.002)$. In controls, the CML immunoreactivity was characterized by numerous weak positive-stained vessels (no difference in the minor (score 1) and moderate (score 2) intensity scores for CML in controls and diabetes), whereas the tissue samples of DM patients showed more pronounced staining (median of the

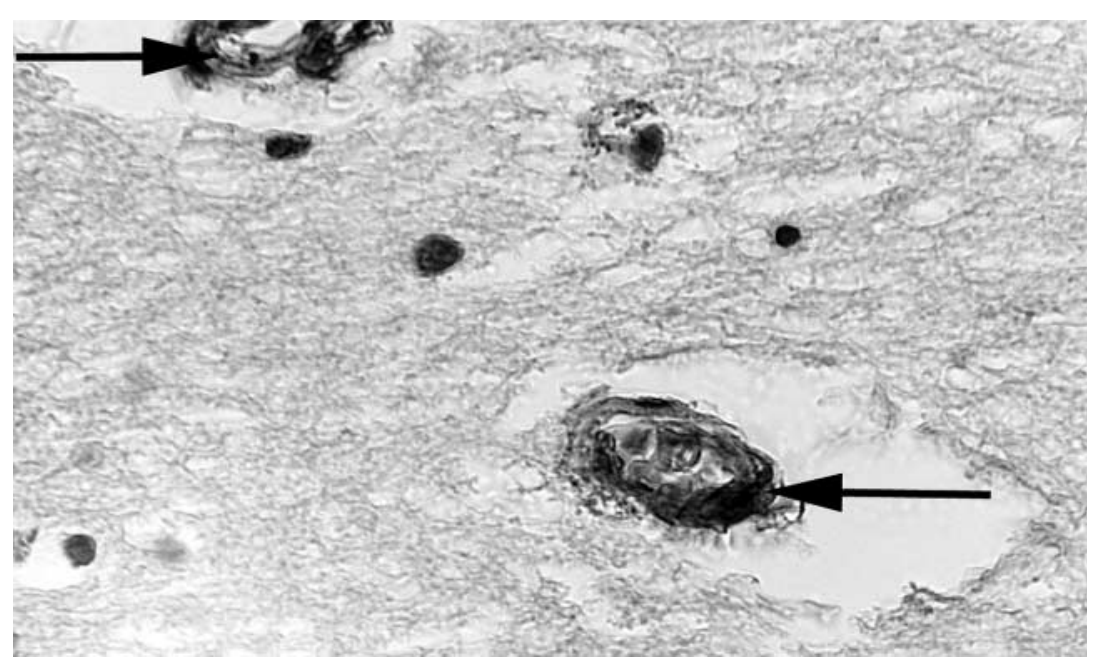

Figure $1 \mathrm{CML}$ staining of human cerebral blood vessels in a patient with diabetes. Arrows indicate CML staining of endothelial cells of cerebral blood vessels. 


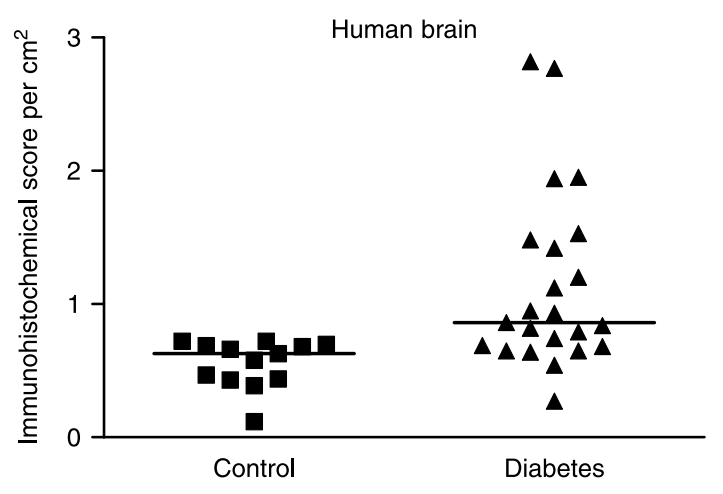

Figure 2 Semiquantative intensity scoring of CML immunoreactivity of cerebral vessels in diabetes patients and age-matched controls. The horizontal lines represent the median of the immunohistochemical score per $\mathrm{cm}^{2}$ (defined in Materials and methods) for CML in control patients (squares) and DM patients (triangles). The difference in immunoreactivity between the control and DM groups was statistically significant $(P=0.002)$.

immunohistochemical intensity score $/ \mathrm{cm}^{2}$ in the DM group of 0.67 (IQR $0.49-1.28$ ) vs 0.29 (IQR $0.18-$ $0.46)$ in the control group; $P=0.002)$, especially in the severe intensity score (score 3) (Table 2). Furthermore, five of the DM patients (four with diabetic nephropathy and one with glomerulosclerosis caused by haemolytic uraemic syndrome) and four of the patients (from which two had end-stage renal disease, one with chronic pyelonephritis and one with nephrolithiasis) in the control group were known with kidney abnormalities. Interestingly, the immunohistochemical intensity scores of the DM patients were around the median. In addition, the kidney function was not statistically different in both groups: the median plasma creatinine level in the DM group was $124 \mu \mathrm{mol} / \mathrm{l}$ (IQR 101-180) and the control group was $140 \mu \mathrm{mol} / \mathrm{l}$ (IQR 75-259), $P=0.87$.

We also evaluated whether CML immunoreactivity correlated with the individual's age. Simple regression analysis, however, found no such association in either group (data not shown).

Table 2 Semiquantative intensity scoring of $N \varepsilon$-(carboxymethyl)lysine (CML) immunoreactivity of human cerebral vessels.

\begin{tabular}{lccc}
\hline & Controls $n=13$ & $\begin{array}{c}\text { Diabetes mellitus } \\
n=20\end{array}$ & $\boldsymbol{P}$ value \\
\hline Immunohistochemical score per cm & & \\
Minor & $0.01(0.00-0.04)$ & $0.00(0.00-0.00)$ & 0.07 \\
Moderate & $0.19(0.13-0.34)$ & $0.19(0.04-0.22)$ & 0.20 \\
Severe & $0.29(0.18-0.46)$ & $0.67(0.49-1.28)$ & $<0.001$
\end{tabular}

Immunohistochemical scores per $\mathrm{cm}^{2}$ for the three different intensity scores for CML (minor, moderate, severe; defined in detail in Materials and methods) are expressed as median (interquartile range, IQR). Significance was determined with the Mann-Whitney test.

\section{Rat tissue}

The immunohistochemical staining observed in the rat model resembles that in human tissue. Again, all the brain tissue slides showed a distinct staining for CML, but with a marked difference in mean immunohistochemical intensity score between STZ-induced diabetic rats and non-diabetic control rats (Fig. 3). Non-diabetic rats showed only minor staining of cerebral vessels. In these rats, the stained vessels were mostly weak positive, with no strong staining of any cerebral vessel (Table 3). The tissue samples of the diabetic rats, however, had a more pronounced staining pattern, with a significant higher score of CML in DM compared with the nondiabetic controls (median of the immunohistochemical intensity score $/ \mathrm{cm}^{2}$ in the diabetic group of 1.08 (IQR $0.73-1.43$ ) vs 0.23 in the control group (IQR $0.12-$ $0.43) ; P=0.003)$.

\section{Discussion}

In this study, we demonstrated the deposition of CMLmodified proteins in the cerebral vessels of diabetic and non-diabetic subjects. The overall intensity of the CML immunoreactivity was significantly increased in the vessels of diabetic patients compared with non-diabetic controls, although some positive staining pattern was also observed in each of the controls. In DM patients, the vessels defined as strongly positive for CML staining (severe/score 3) were more than twofold increased compared with the controls. In a previous study, we had no indication for ongoing oxidation in post-mortem human intramyocardial blood vessels (13). Therefore, we think that the increased CML staining in the DM patients compared with the age-matched control group

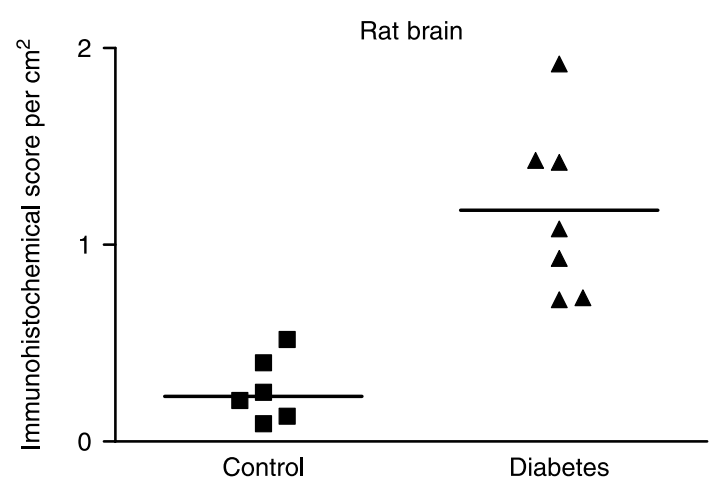

Figure 3 Semiquantative intensity scoring of CML immunoreactivity of cerebral vessels in streptozotocin-induced diabetic rats (DM rats) and non-diabetic controls (non-DM rats). The horizontal lines within the bars represent the median of the immunohistochemical score per $\mathrm{cm}^{2}$ (defined in Materials and methods) for CML in control rats (squares) and DM rats (triangles). The difference in immunoreactivity between the control and DM groups was statistically significant $(P=0.003)$. 
Table 3 Semiquantative intensity scoring of $N \varepsilon$-(carboxymethyl)lysine $(\mathrm{CML})$ immunoreactivity of rat cerebral vessels.

\begin{tabular}{lccc}
\hline & Controls $n=6$ & $\begin{array}{c}\text { Diabetes mellitus } \\
n=7\end{array}$ & $\boldsymbol{P}$ value \\
\hline Immunohistochemical score per cm & & \\
Minor & $0.23(0.08-0.35)$ & $0.53(0.40-0.75)$ & 0.01 \\
Moderate & $0.04(0.00-0.08)$ & $0.40(0.16-0.67)$ & 0.003 \\
Severe & $0.00(0.00-0.00)$ & $0.10(0.00-0.25)$ & 0.036 \\
\hline
\end{tabular}

Immunohistochemical scores per $\mathrm{cm}^{2}$ for the three different intensity scores for CML (minor, moderate, severe; defined in detail in Materials and methods) are expressed as median (interquartile range, IQR). Significance was determined with the Mann-Whitney test.

should be attributed to CML accumulation in the cerebral vasculature during life.

To further explore this finding of CML accumulation, we compared the CML staining intensity of cerebral vessels in STZ-induced diabetic rats and non-diabetic control rats. This model confirmed our findings with human cerebral vessels, as more extensive staining was seen in the cerebral vessels of the diabetic rats. In contrast to the situation in human brain tissues, the rat study showed significant differences in CML immunoreactivity in all three intensity scores for CML.

Previous studies have emphasized a possible link between the accumulation of AGEs in the vessel wall and the diabetic late complications accompanying chronic hyperglycaemia. Indeed, the CML immunoreactivity has been found in the vasculature of the tissues prone to diabetic late complications, such as kidney, retina, heart (13) and peripheral nerves (14). There are several mechanisms by which increased AGE deposition may damage the organs and the vasculature. In general, AGE modification of proteins can alter vascular cell function, interfere with normal cellular interactions and pathologically modify gene expression (15). The consequences of these AGEinduced changes in vascular function are the generation of damaging free radicals, focal thrombosis, impaired vasodilatation, luminal narrowing and excessive vascular inflammation (16).

Several systemic reviews have unequivocally shown that DM is associated with accelerated cognitive decline and dementia $(2,17,18)$. Cognitive impairments have been described in both children and adults, as well as in both type 1 and type 2 diabetes. This DACD may be due to cerebral microvascular damage. Analogous to well-known diabetes-related (micro)vascular complications, such as peripheral diabetic neuropathy and diabetic retinopathy, chronic hyperglycaemia may result from a cascade of events in DACD. Moreover, retinopathy and peripheral neuropathy in type 1 diabetes patients have been shown to be predictive of DACD $(19,20)$. We have shown that type 1 diabetes patients with proliferative retinopathy showed a different cerebral activation pattern, using functional MRI, than those without retinopathy to a cognitive test (21). Reske-Nielsen et al. described, more than 30 years ago in autopsy studies, the changes in the cerebral microvasculature derived from diabetes patients (22). Since then, not much additional data have been published concerning structural abnormalities in the diabetic brain and the cerebral microvasculature.

In our work and in much of the cited studies, it is acknowledged that AGE modification of proteins is relevant to the pathophysiology of these vascular changes. AGE distribution in the vasculature of the kidney, retina and peripheral nerves has extensively been investigated. In addition, Gironès et al. (12) have examined the topographical distribution of CML reactivity in the brain of patients with Alzheimer's disease and diabetes, but they did not quantify this. In our study, we have focused on and quantified the AGE accumulation in the vasculature of the diabetic brain, both in humans and rats. We concluded that DM is associated with excessive accumulation of AGEmodified proteins in the cerebral vasculature. Because vascular function may be adversely affected by AGE, it may be possible that AGEs contribute to the development of cognitive impairments in diabetes.

The cognitive impairment in young and old type 1 diabetes patients is only modest, whereas the cognitive impairment in older type 2 diabetes patients is more pronounced $(17,23)$. This might be due to differential effects of type 1 and type 2 diabetes on the brain.

The major limitation of our study is that no relation has been made between the intensity of AGE accumulation and the severity of the cognitive decline. Therefore, the future studies with a rat model of (type 1 or 2 ) diabetes should focus on cognitive function and the association with AGE accumulation. The data reported here emphasize the need of further study into a causal link between AGE accumulation in cerebral vessels and cognitive dysfunction. Understanding the contribution of AGEs should provide insight into the complex aetiology of any diabetes-related cognitive disorder. Moreover, it is clear that studies should be developed to find out the effect of AGE inhibitors as aminoguanidine (24) on AGE accumulation in cerebral vessels and cognitive impairment.

\section{References}

1 Standards of medical care in diabetes - 2006. Diabetes Care 2006 29 S4-S42.

2 Arvanitakis Z, Wilson RS \& Bennett DA. Diabetes mellitus, dementia, and cognitive function in older persons. Journal of Nutrition, Health $\mathcal{E}$ Aging 200610 287-291.

3 Mijnhout GS, Scheltens P, Diamant M, Biessels GJ, Wessels AM, Simsek S, Snoek FJ \& Heine RJ. Diabetic encephalopathy: a concept in need of a definition. Diabetologia $2006 \mathbf{4 9} 1447-1448$.

4 Toth C, Schmidt AM, Tuor UI, Francis G, Foniok T, Brussee V, Kaur J, Yan SF, Martinez JA, Barber PA, Buchan A \& Zochodne DW. Diabetes, leukoencephalopathy and rage. Neurobiology of Disease 200623 445-461.

5 Brownlee M. Advanced protein glycosylation in diabetes and aging. Annual Review of Medicine 199546 223-234. 
6 Furth AJ. Glycated proteins in diabetes. British Journal of Biomedical Science 199754 192-200.

7 McCance DR, Dyer DG, Dunn JA, Bailie KE, Thorpe SR, Baynes JW \& Lyons TJ. Maillard reaction products and their relation to complications in insulin-dependent diabetes mellitus. Journal of Clinical Investigations 199391 2470-2478.

8 Genuth S, Sun W, Cleary P, Sell DR, Dahms W, Malone J, Sivitz W \& Monnier VM. Glycation and carboxymethyllysine levels in skin collagen predict the risk of future 10-year progression of diabetic retinopathy and nephropathy in the diabetes control and complications trial and epidemiology of diabetes interventions and complications participants with type 1 diabetes. Diabetes 2005 54 3103-3111.

9 Hughes JM, Kuiper EJ, Klaassen I, Canning P, Stitt AW, Van Bezu J, Schalkwijk CG, Van Noorden CJF \& Schlingemann RO. Advanced glycation end products cause increased CCN family and extracellular matrix gene expression in the diabetic rodent retina. Diabetologia 200750 1089-1098.

10 Schalkwijk CG, Baidoshvili A, Stehouwer CD, van Hinsbergh VW \& Niessen HW. Increased accumulation of the glycoxidation product Ne-(carboxymethyl)lysine in hearts of diabetic patients: generation and characterisation of a monoclonal anti-CML antibody. Biochimica et Biophysica Acta 20041636 82-89.

11 Miki HC, Nagai R, Miyazaki K, Hayase F, Araki T, Ono T \& Horiuchi S. Conversion of Amadori products of the Maillard reaction to NE-(carboxymethyl)lysine by short-term heating: possible detection of artifacts by immunohistochemistry. Laboratory Investigation 2002 82 795-808.

12 Girones X, Guimera A, Cruz-Sanchez CZ, Ortega A, Sasaki N, Makita Z, Lafuente JV, Kalaria R \& Cruz-Sanchez FF. NEcarboxymethyllysine in brain aging, diabetes mellitus, and Alzheimer's disease. Free Radical Biology \& Medicine 200436 1241-1247.

13 Baidoshvilli A, Krijnen PAJ, Kupreishvili K, Ciurana C, Bleeker W, Nijmeijer R, Visser CA, Visser FC, Meijer CJLM, Stooker W, Eijsman L, van Hinsbergh VWM, Hack CE, Niessen HWM \& Schalkwijk CG. Ne-(carboxymethyl)lysine depositions in intramyocardial blood vessels in human and rat acute myocardial infarction: a predictor or reflection of infarction? Arteriosclerosis, Thrombosis, and Vascular Biology 200626 2497-2503.
14 Wada R \& Yagihashi S. Role of advanced glycation end products and their receptors in development of diabetic neuropathy. Annals of New York Academy of Sciences 20051043 598-604.

15 Brownlee M. Negative consequences of glycation. Metabolism 200049 9-13.

16 Basta G, Schmidt AM \& De CR. Advanced glycation end products and vascular inflammation: implications for accelerated atherosclerosis in diabetes. Cardiovascular Research 200463 582-592.

17 Brands AM, Biessels GJ, de Haan EH, Kappelle LJ \& Kessels RP. The effects of type 1 diabetes on cognitive performance: a metaanalysis. Diabetes Care 200528 726-735.

18 Strachan MW, Deary IJ, Ewing FM \& Frier BM. Is type II diabetes associated with an increased risk of cognitive dysfunction? A critical review of published studies Diabetes Care 199720 $438-445$.

19 Ryan CM, Williams TM, Orchard TJ \& Finegold DN. Psychomotor slowing is associated with distal symmetrical polyneuropathy in adults with diabetes mellitus. Diabetes 199241 107-113.

20 Ferguson SC, Blane A, Perros P, McCrimmon RJ, Best JJ, Wardlaw J, Deary IJ \& Frier BM. Cognitive ability and brain structure in type 1 diabetes: relation to microangiopathy and preceding severe hypoglycemia. Diabetes 200352 149-156.

21 Wessels AM, Rombouts SARB, Simsek S, Kuijer JPA, Kostense PJ, Barkhof F, Scheltens Ph, Snoek FJ \& Heine RJ. Microvascular disease in type 1 diabetes alters brain activation: a functional MRI study. Diabetes 200655 334-340.

22 Reske-Nielsen E, Lundbaek K \& Rafaelsen OJ. Pathological changes in the central and peripheral nervous system of young longterm diabetics. Diabetologia 19651 233-241.

23 Manschot SM, Brands AMA, van der Grond J, Kessels RP, Algra A, Kappelle LJ \& Biessels GJ. Brain magnetic resonance imaging correlates of impaired cognition in patients with type 2 diabetes. Diabetes 200655 1106-1113.

24 Huijberts MS \& Schalkwijk CG. Is AGE accumulation a therapeutic target for diabetic complications? Netherlands Journal of Medicine $2006641-3$.

Received 29 January 2008

Accepted 11 February 2008 